\title{
A VARIATIONAL INEQUALITY IN NON-COMPACT SETS AND ITS APPLICATIONS
}

\author{
WON KYU KIM AND KoK-KeONg TAN
}

In this note, we shall prove a new variational inequality in non-compact sets and as an application, we prove a generalisation of the Schauder-Tychonoff fixed point theorem.

Let $E$ be a Hausdorff topological vector space. Denote the dual space of $E$ by $E^{*}$ and the pairing between $E^{*}$ and $E$ by $\langle w, x\rangle$ for each $w \in E^{*}$ and $x \in E$. If $A$ is a subset of $E$, we shall denote by $2^{A}$ the family of all non-empty subsets of $A$ and by cl $A$ the closure of $A$ in $E$, and $\operatorname{co} A$ the convex hull of $A$.

The following Fan-Browder fixed point theorem [2] is essential in convex analysis and is also the basic tool in proving many variational inequalities and intersection theorems in nonlinear functional analysis:

ThEOREM. [2] Let $X$ be a non-empty compact convex subset of a Hausdorff topological vector space and let $T: X \rightarrow 2^{X}$ be a multimap satisfying the following:

(1) for each $x \in X, T(x)$ is convex,

(2) for each $y \in X, T^{-1}(y)$ is open.

Then $T$ has a fixed point $\widehat{x} \in X$, that is $\widehat{x} \in T(\widehat{x})$.

The Fan-Browder theorem can be proved by using Brouwer's fixed point theorem or the KKM-theorem. Until now, there have been numerous generalisations and applications of this Theorem by several authors; for example, see $[4,7]$ and the references there.

In a recent paper [4], Ding, Kim and Tan further generalise the above result to non-compact sets in locally convex spaces and the following is a special case of the fixed point version of their Theorem 1:

LEMMA 1. [4] Let $X$ be a non-empty convex subset of a locally convex Hausdorff topological vector space and $D$ be a non-empty compact subset of $X$. Let $T: X \rightarrow 2^{D}$ be a multimap satisfying the following:

(1) for each $x \in X, \operatorname{co} T(x) \subset D$,

(2) for each $y \in X, T^{-1}(y)$ is open in $X$.

Received 15 August, 1991

This paper was partially supported by NSERC of Canada under grant A-8096, and for the first author by a grant from the Korea Science and Engineering Foundation in 1992.

Copyright Clearance Centre, Inc. Serial-fee code: 0004-9729/92 $\$ A 2.00+0.00$. 
Then there exists a point $\widehat{x} \in X$ such that $\widehat{x} \in \operatorname{co} T(\widehat{x})$.

Recall that for a topological vector space $E$, the strong topology on its dual space $E^{*}$ is the topology on $E^{*}$ generated by the family $\{U(B ; \varepsilon): B$ is a non-empty bounded subset of $E$ and $E>0\}$ as a base for the neighbourhood system at 0 , where $U(B ; \varepsilon)=$ $\left\{f \in E^{*}: \sup _{x \in B}|\langle f, x\rangle|<\varepsilon\right\}$.

We begin with the following

LEMMA 2. Let $E$ be a topological vector space and $E^{*}$ be the dual space of $E$ equipped with the strong topology. Let $X$ be a non-empty bounded subset of $E$ and $T: X \rightarrow 2^{E^{*}}$ be an upper semicontinuous multimap such that each $T(x)$ is (strongly) compact. Then for each $y \in E$, the real-valued function $g_{y}: X \rightarrow R$ defined by

$$
g_{y}(x)=\inf _{w \in T(x)} \operatorname{Re}\langle w, x-y\rangle, \quad \text { for each } x \in X
$$

is lower semicontinuous.

Proof: Let $x_{0} \in X$ be given. For any $\varepsilon>0$, we shall show that there exists an open neighbourhood $N\left(x_{0}\right)$ of $x_{0}$ such that

$$
g_{y}(x) \geqslant g_{y}\left(x_{0}\right)-\epsilon \quad \text { for each } x \in N\left(x_{0}\right)
$$

Indeed, let $V:=\left\{p \in E^{*}: \sup _{t \in X-y}|p(t)|<\varepsilon / 3\right\}$, where $X-y=\{x-y: x \in X\}$. Then $V$ is a strongly open neighbourhood of 0 in $E^{*}$ since $X-y$ is a bounded set in $E$. Since $T$ is upper semicontinuous at $x_{0}$ and $T\left(x_{0}\right)+V$ is a strongly open set containing $T\left(x_{0}\right)$, there exists an open neighbourhood $N_{0}$ of $x_{0}$ in $X$ such that $T(x) \subset T\left(x_{0}\right)+V$ for each $x \in N_{0}$.

Next, for each $u \in T\left(x_{0}\right)$, we let

$$
V_{u}:=\left\{p \in E^{*}: \sup _{t \in X-X}|p(t)-u(t)|<\frac{\varepsilon}{3}\right\}
$$

where $X-X=\{x-z: x, z \in X\}$; then $V_{u}$ is also a strongly open neighbourhood of $u$ in $E^{*}$ since $X-X$ is a bounded set in $E$. Since $T\left(x_{0}\right)$ is strongly compact and $T\left(x_{0}\right) \subset \cup_{u \in T\left(x_{0}\right)} V_{u}$, there exists a finite subset $\left\{u_{1}, \ldots, u_{n}\right\}$ of $T\left(x_{0}\right)$ with $T\left(x_{0}\right) \subset$ $\cup_{i=1}^{n} V_{u_{i}}$. For each $i=1, \ldots, n$, since $u_{i}$ is a continuous linear functional, there exists an open neighbourhood $N_{i}$ of $x_{0}$ in $X$ such that $\left|u_{i}(x)-u_{i}\left(x_{0}\right)\right|<\varepsilon / 3$ for each $x \in N_{i}$.

Now let $N\left(x_{0}\right):=\bigcap_{i=0}^{n} N_{i}$; then $N\left(x_{0}\right)$ is an open neighbourhood of $x_{0}$ in $X$. We shall show that this open neighbourhood $N\left(x_{0}\right)$ of $x_{0}$ is the desired one. For each $x \in N\left(x_{0}\right)$ and each $w \in T(x)$, since $x \in N_{0}$, there exists $u \in T\left(x_{0}\right)$ such 
that $w-u \in V$. Also, since $u \in T\left(x_{0}\right) \subset \cup_{i=1}^{n} V_{u_{i}}$, there exists $i_{0} \in\{1, \ldots, n\}$ such that $u \in V_{u_{i_{0}}}$. Therefore we have

$$
|R e\langle w, x-y\rangle-\operatorname{Re}\langle u, x-y\rangle| \leqslant|\langle w-u, x-y\rangle|<\frac{\varepsilon}{3},
$$

so that

$$
\begin{aligned}
\operatorname{Re}\langle w, x-y\rangle> & \operatorname{Re}\langle u, x-y\rangle-\frac{\varepsilon}{3} \\
= & \operatorname{Re}\left\langle u, x_{0}-y\right\rangle+\operatorname{Re}\left\langle u, x-x_{0}\right\rangle-\frac{\varepsilon}{3} \\
= & \operatorname{Re}\left\langle u, x_{0}-y\right\rangle+\operatorname{Re}\left\langle u-u_{i_{0}}, x-x_{0}\right\rangle \\
& \quad+\operatorname{Re}\left\langle u_{i_{0}}, x-x_{0}\right\rangle-\frac{\varepsilon}{3} \\
> & \operatorname{Re}\left\langle u, x_{0}-y\right\rangle-\frac{\varepsilon}{3}-\frac{\varepsilon}{3}-\frac{\varepsilon}{3} \\
\geqslant & \inf _{v \in T\left(x_{0}\right)} \operatorname{Re}\left\langle v, x_{0}-y\right\rangle-\varepsilon \\
= & g_{y}\left(x_{0}\right)-\varepsilon .
\end{aligned}
$$

Since $w \in T(x)$ is arbitrary, we have $g_{y}(x)=\inf _{w \in T(x)} R e\langle w, x-y\rangle \geqslant g_{y}\left(x_{0}\right)-\varepsilon$, which completes the proof.

Lemma 2 is a multivalued generalisation of Lemma 1 in [2] (see also [10, Lemma 1] where it was observed that the result holds for $X$ being bounded instead of compact).

Now we shall prove the following new variational inequality in non-compact sets.

Theorem 1. Let $X$ be a bounded convex subset of a locally convex Hausdorff topological vector space $E$ and $D$ be a non-empty compact subset of $X$. Let $T$ : $X \rightarrow 2^{E^{*}}$ be an upper semicontinuous multimap from the relative topology of $X$ to the strong topology of $E^{*}$ such that each $T(x)$ is (strongly) compact. Suppose further that for each $x \in X \backslash D$,

$$
\inf _{w \in T(y)} \operatorname{Re}\langle w, y-x\rangle \leqslant 0 \quad \text { for all } y \in X .
$$

Then there exists a point $\widehat{x} \in X$ such that

$$
\inf _{w \in T(\widehat{x})} \operatorname{Re}(w, \widehat{x}-x) \leqslant 0 \quad \text { for all } x \in X .
$$

Furthermore, if $T(\widehat{x})$ is also convex, then there exists a point $\widehat{w} \in T(\widehat{x})$ such that

$$
R e\langle\widehat{w}, \widehat{x}-x\rangle \leqslant 0 \quad \text { for all } x \in X
$$


Proof: Suppose that for each $x \in X$ there exists a point $\widetilde{x} \in X$ such that $\inf _{w \in T(x)} \operatorname{Re}\langle w, x-\tilde{x}\rangle>0$. Then by the assumption $(*), \tilde{x} \in D$. Now we define a multimap $P: X \rightarrow 2^{D}$ by

$$
P(x):=\left\{y \in D: \inf _{w \in T(x)} R e\langle w, x-y\rangle>0\right\} \quad \text { for all } x \in X
$$

Then for each $x \in X, P(x)$ is non-empty. For each $x \in X$, we shall show that $\operatorname{co} P(x) \subset$ $D$. Indeed, let $n \in N, y_{1}, \ldots, y_{n} \in P(x)$ and $t_{1}, \ldots, t_{n} \in[0,1]$ with $\sum_{i=1}^{n} t_{i}=1$; then for each $i=1, \ldots, n$,

$$
\inf _{w \in T(x)} \operatorname{Re}\left\langle w, x-y_{i}\right\rangle>0
$$

it follows that

$$
\inf _{w \in T(x)} \operatorname{Re}\left\langle w, x-\sum_{i=1}^{n} t_{i} y_{i}\right\rangle \geqslant \sum_{i=1}^{n} t_{i} \inf _{w \in T(x)} R e\left\langle w, x-y_{i}\right\rangle>0 .
$$

Since $\sum_{i=1}^{n} t_{i} y_{i} \in X$, by the assumption $(*)$ again, $\sum_{i=1}^{n} t_{i} y_{i} \in D$. Hence $c o P(x) \subset D$.

Next for each $y \in D$, we shall show that $P^{-1}(y)$ is open in $X$. Let $\left(x_{\alpha}\right)_{\alpha \in \Gamma}$ be a net in $X \backslash P^{-1}(y)$, which converges to some $x_{0} \in X$. Then we have

$$
\inf _{w \in T\left(x_{\alpha}\right)} R e\left\langle w, x_{\alpha}-y\right\rangle \leqslant 0 \quad \text { for all } \alpha \in \Gamma
$$

By Lemma 2, the real-valued function

$$
x \rightarrow \inf _{w \in T(x)} \operatorname{Re}\langle w, x-y\rangle
$$

is lower semicontinuous, it follows that

$$
\inf _{w \in T\left(x_{0}\right)} R e\left\langle w, x_{0}-y\right\rangle \leqslant 0 .
$$

Therefore $X \backslash P^{-1}(y)$ is closed, and hence $P^{-1}(y)$ is open in $X$. Thus all the hypotheses of Lemma 1 are satisfied, so that by Lemma 1 there exists a point $\widehat{x} \in X$ such that $\widehat{x} \in \operatorname{co} P(\widehat{x})$. But then there exist $y_{1}, \ldots, y_{m} \in P(\widehat{x})$ and $\lambda_{1}, \ldots, \lambda_{m} \geqslant 0$ with $\sum_{i=1}^{m} \lambda_{i}=$ 1 such that $\widehat{x}=\sum_{i=1}^{m} \lambda_{i} y_{i}$. Therefore we have 


$$
\begin{aligned}
0 & =\inf _{w \in T(\hat{x})} \operatorname{Re}\langle w, \widehat{x}-\widehat{x}\rangle \\
& =\inf _{w \in T(\hat{x})} \operatorname{Re}\left\langle w, \widehat{x}-\sum_{i=1}^{m} \lambda_{i} y_{i}\right\rangle \\
& =\inf _{w \in T(\widehat{x})} \sum_{i=1}^{m} \lambda_{i} \operatorname{Re}\left\langle w, \widehat{x}-y_{i}\right\rangle \\
& \geqslant \sum_{i=1}^{m} \lambda_{i} \inf _{w \in T(\widehat{x})} \operatorname{Re}\left\langle w, \widehat{x}-y_{i}\right\rangle>0,
\end{aligned}
$$

which is a contradiction. Hence there must exist a point $\widehat{x} \in X$ such that

$$
\inf _{w \in T(\widehat{x})} \operatorname{Re}\langle w, \widehat{x}-x\rangle \leqslant 0 \quad \text { for all } x \in X
$$

To prove the second assertion, suppose that $T(\widehat{x})$ is convex. Then we define $f$ : $X \times T(\widehat{x}) \rightarrow R$ by

$$
f(x, w):=\operatorname{Re}\langle w, \widehat{x}-x\rangle \text { for each }(x, w) \in X \times T(\widehat{x}) .
$$

Note that for each fixed $x \in X, x \rightarrow R e\langle w, \widehat{x}-x\rangle$ is continuous affine, and for each $w \in T(\widehat{x}), x \rightarrow R e\langle w, \widehat{x}-x\rangle$ is affine. Thus, by Kneser's minimax theorem [8], we have

Thus

$$
\min _{w \in T(\widehat{x})} \sup _{x \in X} f(x, w)=\sup _{x \in X} \min _{w \in T(\widehat{x})} f(x, w) \text {. }
$$

$$
\min _{w \in T(\widehat{x})} \sup _{x \in X} \operatorname{Re}\langle w, \widehat{x}-x\rangle \leqslant 0 \quad \text { by }(1)
$$

Since $T(\widehat{x})$ is compact, there exists $\widehat{w} \in T(\widehat{x})$ such that

$$
\sup _{x \in X} \operatorname{Re}\langle\widehat{w}, \widehat{x}-x\rangle=\min _{w \in T(\widehat{x})} \sup _{x \in X} \operatorname{Re}\langle w, \widehat{x}-x\rangle
$$

Therefore $\operatorname{Re}\langle\widehat{w}, \widehat{x}-x\rangle \leqslant 0$ for all $x \in X$. This completes the proof.

When $X=D$ is compact convex, we obtain the following generalisation of Hartman-Stampacchia's variational inequality [6] due to Browder [3, Theorem 6]:

Corollary 1 . Let $X$ be a non-empty compact convex subset of a locally convex Hausdorff topological vector space $E$ and let $T: X \rightarrow 2^{E^{*}}$ be an upper semicontinuous multimap from the relative topology of $X$ to the strong topology of $E^{*}$ such that each $T(x)$ is a (strongly) compact convex subset of $E^{*}$. 
Then there exists a point $\widehat{x} \in X$ and $\widehat{w} \in T(\widehat{x})$ such that

$$
\operatorname{Re}\langle\widehat{w}, \widehat{x}-x\rangle \leqslant 0 \quad \text { for all } x \in X
$$

The following is a single-valued version of Theorem 1:

Corollary 2. Let $X$ be a bounded convex subset of a locally convex Hausdorff topological vector space $E$ and $D$ be a non-empty compact subset of $X$. Let $T: X \rightarrow$ $E^{*}$ be a continuous mapping from the relative topology of $X$ to the strong topology of $E^{*}$ satisfying the following condition:

$$
\text { for each } x \in X \backslash D, \operatorname{Re}\langle T(y), y-x\rangle \leqslant 0 \quad \text { for all } y \in X \text {. }
$$

Then there exists a point $\widehat{x} \in X$ such that

$$
\operatorname{Re}\langle T(\widehat{x}), \widehat{x}-x\rangle \leqslant 0 \quad \text { for all } x \in X
$$

Let $E$ be a topological vector space and $M$ be a topological space. Recall that a multimap $F: M \rightarrow 2^{E}$ is upper hemicontinuous (for example, see $[1, p .122]$ ) if for each $p \in E^{*}$ and for each $\lambda \in R$, the set $\left\{x \in M: \sup _{u \in F(x)} \operatorname{Re}\langle p, u\rangle<\lambda\right\}$ is open in $M$. For relationships among upper semicontinuity, upper demicontinuity and upper hemicontinuity, we refer to [11, Propositions 1 and 2 and Examples 1 and 2].

As an application of Corollary 2, we prove the following fixed point theorem:

THEOREM 2. Let $X$ be a non-empty paracompact bounded convex subset of a locally convex Hausdorff topological vector space $E, D$ be a non-empty compact subset of $X$. Let $F: X \rightarrow 2^{E}$ be an upper hemicontinuous multimap satisfying the following:

(1) for each $x \in X, F(x)$ is non-empty closed convex,

(2) for each $x \in X, F(x) \cap \operatorname{cl}\left(x+\cup_{\lambda>0} \lambda(X-x)\right) \neq \phi$,

(3) for each $x \in X \backslash D, y \in X$ and $p \in E^{*}$, if $\inf \{\operatorname{Re}\langle p, y-z\rangle: z \in F(y)\}>$ 0 , then $\operatorname{Re}\langle p, y-x\rangle \leqslant 0$.

Then there exists $\widehat{x} \in X$ such that $\widehat{x} \in F(\widehat{x})$.

ProOF: Since $F$ is upper hemicontinuous, for each $p \in E^{*}$, the set

$$
\begin{gathered}
U(p)=\left\{x \in X: \sup _{z \in F(x)} \operatorname{Re}\langle p, z\rangle<\operatorname{Re}\langle p, x\rangle\right\} \\
=\cup_{\lambda \in R}\left[\left\{x \in X: \sup _{z \in F(x)} \operatorname{Re}\langle p, z\rangle<\lambda\right\}\right. \\
\cap\{x \in X: \operatorname{Re}\langle p, x\rangle>\lambda\}]
\end{gathered}
$$

is open in $X$. Suppose $x \notin F(x)$ for each $x \in X$. Then for each $x \in X$, there exists $p \in E^{*}$ such that $\sup _{z \in F(x)} \operatorname{Re}\langle p, z\rangle<\operatorname{Re}\langle p, x\rangle$ so that $x \in U(p)$. Thus $\left\{U(p): p \in E^{*}\right\}$ 
is an open cover of the paracompact space $X$. Let $\left\{V(p): p \in E^{*}\right\}$ be a locally finite open precise refinement of $\left\{U(p): p \in E^{*}\right\}$ and $\left\{\beta_{p}: p \in E^{*}\right\}$ be the continuous partition of unity subordinated to this refinement $\left\{V(p): p \in E^{*}\right\}$. Define a mapping $T: X \rightarrow E^{*}$ by

$$
T(x)=\sum_{p \in E^{*}} \beta_{p}(x) p \quad \text { for all } x \in X .
$$

Let $x \in X$ be given. If $p \in E^{*}$ and $\beta_{p}(x)>0$, then $x \in V(p) \subset U(p)$ so that $\sup _{z \in F(x)} \operatorname{Re}\langle p, z\rangle<\operatorname{Re}\langle p, x\rangle ;$ it follows that $\inf _{z \in F(x)} \operatorname{Re}\langle p, x-z\rangle>0$. Therefore for each $x \in X$,

$$
\begin{aligned}
\inf _{z \in F(x)} \operatorname{Re}\langle T(x), x-z\rangle & =\inf _{z \in F(x)} \sum_{p \in E^{*}} \beta_{p}(x) \operatorname{Re}\langle p, x-z\rangle \\
& \geqslant \sum_{p \in E^{*}} \beta_{p}(x) \inf _{z \in F(x)} \operatorname{Re}\langle p, x-z\rangle \\
& >0 .
\end{aligned}
$$

Now we shall show that $T$ satisfies all hypotheses of Corollary 2. To show that $T$ is continuous from the relative topology of $X$ to the strong topology of $E^{*}$, let $\left(x_{\alpha}\right)_{\alpha \in \Gamma}$ be a net in $X$ which converges to some $x_{0} \in X$. Since $\left\{V(p): p \in E^{*}\right\}$ is locally finite, there is an open neighbourhood $U_{0}$ of $x_{0}$ in $X$ such that $\left\{p \in E^{*}: V(p) \cap U_{0} \neq \phi\right\}$ is finite, so we let $\left\{p \in E^{*}: V(p) \cap U_{0} \neq \phi\right\}=\left\{p_{1}, \ldots, p_{n}\right\}$. Let $B$ be any non-empty bounded subset of $E$; then by Theorem $1.18[9], M=\max _{1 \leqslant i \leqslant n} \sup \left\{\left|p_{i}(x)\right|: x \in B\right\}<\infty$. Since each $\beta_{p_{i}}$ is continuous, there exists $\alpha_{1} \in \Gamma$ such that for each $\alpha \geqslant \alpha_{1}$,

$$
\left|\beta_{p_{i}}\left(x_{\alpha}\right)-\beta_{p_{i}}\left(x_{0}\right)\right|<\frac{\varepsilon}{M n} \text { for all } i=1, \ldots, n \text {. }
$$

Also since $\left(x_{\alpha}\right)$ converges to $x_{0}$ and $U_{0}$ is an open neighbourhood of $x_{0}$, there exists $\alpha_{2} \in \Gamma$ such that for each $\alpha \geqslant \alpha_{2}, x_{\alpha} \in U_{0}$. Let $\alpha_{0} \geqslant \max \left\{\alpha_{1}, \alpha_{2}\right\}$. Then for each $\alpha \geqslant \alpha_{0}$, we have

$$
\begin{aligned}
& \sup _{z \in B}\left|\left\langle T\left(x_{\alpha}\right)-T\left(x_{0}\right), z\right\rangle\right| \\
= & \sup _{z \in B}\left|\sum_{p \in E^{*}}\left(\beta_{p}\left(x_{\alpha}\right)-\beta_{p}\left(x_{0}\right)\right) p(z)\right| \\
= & \sup _{z \in B}\left|\sum_{i=1}^{n}\left(\beta_{p_{i}}\left(x_{\alpha}\right)-\beta_{p_{i}}\left(x_{0}\right)\right) p_{i}(z)\right| \\
\leqslant & \sum_{i=1}^{n}\left|\left(\beta_{p_{i}}\left(x_{\alpha}\right)-\beta_{p_{i}}\left(x_{0}\right)\right)\right| \sup _{z \in B}\left|p_{i}(z)\right| \\
< & \sum_{i=1}^{n} \frac{\varepsilon}{M n} M=\varepsilon,
\end{aligned}
$$


and hence $\left(T\left(x_{\alpha}\right)\right)$ converges to $T\left(x_{0}\right)$ in the strong topology of $E^{*}$.

Next suppose there exists $x_{1} \in X \backslash D$ such that for some $y \in X$,

$$
\operatorname{Re}\left\langle T(y), y-x_{1}\right\rangle=\sum_{p \in E^{*}} \beta_{p}(y) \operatorname{Re}\left\langle p, y-x_{1}\right\rangle>0 .
$$

If $\beta_{p}(y)>0$, then $\inf _{z \in F(y)} \operatorname{Re}\langle p, y-z\rangle>0$ so that by (3), $\operatorname{Re}\left\langle p, y-x_{1}\right\rangle \leqslant 0$, which contradicts $(* *)$.

Therefore by Corollary 2, there exists $\widehat{x} \in X$ such that

$$
\operatorname{Re}\langle T(\widehat{x}), \widehat{x}-y\rangle \leqslant 0 \text { for all } y \in X .
$$

By the assumption (2), $F(\widehat{x}) \cap \operatorname{cl}\left(\widehat{x}+\cup_{\lambda>0} \lambda(X-\widehat{x})\right) \neq \phi$. Let $\widehat{y} \in F(\widehat{x}),\left(\lambda_{\alpha}\right)_{\alpha \in \Gamma}$ be a net in $(0, \infty)$ and $\left(u_{\alpha}\right)_{\alpha \in \Gamma}$ be a net in $X$ such that $\left(\widehat{x}+\lambda_{\alpha}\left(u_{\alpha}-\widehat{x}\right)\right) \rightarrow \widehat{y}$. Then we have

$$
\begin{aligned}
\operatorname{Re}\langle T(\widehat{x}), \widehat{x}-\widehat{y}\rangle & =\lim _{\alpha} \operatorname{Re}\left\langle T(\widehat{x}), \widehat{x}-\left(\widehat{x}+\lambda_{\alpha}\left(u_{\alpha}-\widehat{x}\right)\right)\right\rangle \\
& =\lim _{\alpha} \lambda_{\alpha} \operatorname{Re}\left\langle T(\widehat{x}), \widehat{x}-u_{\alpha}\right\rangle \\
& \leqslant 0 \quad \text { by }(* * *) .
\end{aligned}
$$

Hence $\inf _{z \in F(\widehat{x})} \operatorname{Re}\langle T(\widehat{x}), \widehat{x}-z\rangle \leqslant 0$, which contradicts $(*)$. This completes the proof. $\square$

Theorem 2 generalises Theorem 2 of Halpern $[5, \mathrm{p} .88]$ in the following ways: (i) $X$ need not be compact and (ii) $F$ is upper hemicontinuous instead of upper semicontinuous.

The following is a reformulation of Proposition 3.1.21 of Aubin-Ekeland [1]:

Lemma 3. Let $X$ and $Y$ be topological spaces and $\Phi: X \times Y \rightarrow R$ be a real-valued lower semicontinuous function on $X \times Y$ and $T: Y \rightarrow 2^{X}$ be upper semicontinuous at $y_{0} \in Y$ and $T\left(y_{0}\right)$ is non-empty compact. Then a real-valued function $g: Y \rightarrow R$ defined by

$$
g(y):=\inf _{x \in T(y)} \Phi(x, y), \quad \text { for all } y \in Y
$$

is lower semicontinuous at $y_{0}$.

Lemma 4. Let $E$ be a normed space, $X$ be a non-empty subset of $E$ and $T: X \rightarrow 2^{E^{*}}$ be an upper semicontinuous multimap such that each $T(x)$ is (norm-) compact. Then for each $y \in E$, the real-valued function $g_{y}: X \rightarrow R$ defined by

$$
g_{y}(x):=\inf _{w \in T(x)} R e\langle w, x-y\rangle, \quad \text { for each } x \in X,
$$


is lower semicontinuous.

Proof: Define $\Phi: X \times E^{*} \rightarrow R$ by

$$
\Phi(x, w)=R e\langle w, x-y\rangle \quad \text { for each }(x, w) \in X \times E^{*}
$$

Let $\left(x_{n}\right)$ be a sequence in $X$ which converges to $x \in X$ and $\left(w_{n}\right)$ be a sequence in $E^{*}$ which converges to $w \in E^{*}$. Then we have

$$
\begin{aligned}
& \left|\Phi\left(x_{n}, w_{n}\right)-\Phi(x, w)\right| \\
= & \left|R e\left\langle w_{n}, x_{n}-y\right\rangle-\operatorname{Re}\langle w, x-y\rangle\right| \\
\leqslant & \left|\left\langle w_{n}-w, x-y\right\rangle\right|+\left|\left\langle w_{n}, x_{n}-x\right\rangle\right| \\
\leqslant & \left\|w_{n}-w\right\|\|x-y\|+\left\|w_{n}\right\|\left\|x_{n}-x\right\| \rightarrow 0,
\end{aligned}
$$

since $\left\{\left\|w_{n}\right\|: n \geqslant 1\right\}$ is bounded.

Thus $\Phi$ is continuous. By Lemma 3, $g_{y}$ is lower semicontinuous. This completes the proof.

We remark that in the proof of Theorem 1 , the condition " $X$ is bounded" was never needed until Lemma 2 was quoted. In view of Lemma 4, the same proof of Theorem 1 gives the following

ThEOREM 3. Let $X$ be a convex subset of a normed linear space $E$ and $D$ be a non-empty compact subset of $X$. Let $T: X \rightarrow 2^{E^{*}}$ be an upper semicontinuous multimap from the relative topology of $X$ to the norm topology of $E^{*}$ such that each $T(x)$ is (norm-) compact in $E^{*}$. Suppose further that for each $x \in X \backslash D$,

$$
\inf _{w \in T(y)} \operatorname{Re}\langle w, y-x\rangle \leqslant 0 \quad \text { for all } y \in X
$$

Then there exists a point $\widehat{x} \in X$ such that

$$
\inf _{w \in T(\widehat{x})} R e\langle w, \widehat{x}-x\rangle \leqslant 0 \quad \text { for all } x \in X
$$

Furthermore, if $T(\widehat{x})$ is also convex, then there exists a point $\widehat{w} \in T(\widehat{x})$ such that

$$
\operatorname{Re}\langle\widehat{w}, \widehat{x}-x\rangle \leqslant 0 \quad \text { for all } x \in X .
$$

For the same reason, in case $E$ is a normed space, the condition that $X$ be bounded in Corollary 2 can be deleted. As a result, we have the following norm-version of Theorem 2; recalling that every metric space is paracompact: 
ThEOREM 4. Let $X$ be a non-empty convex subset of a normed linear space $E$ and $D$ be a non-empty compact subset of $X$. Let $F: X \rightarrow 2^{E}$ be an upper hemicontinuous multimap satisfying the following:

(1) for each $x \in X, F(x)$ is non-empty closed convex,

(2) for each $x \in X, F(x) \cap c l\left(x+\cup_{\lambda>0} \lambda(X-x)\right) \neq \phi$,

(3) for each $x \in X \backslash D, y \in X$ and $p \in E^{*}$, if $\inf \{\operatorname{Re}\langle p, y-z\rangle: z \in F(y)\}>$ 0 , then $\operatorname{Re}\langle p, y-x\rangle \leqslant 0$.

Then there exists $\widehat{x} \in X$ such that $\widehat{x} \in F(\widehat{x})$.

\section{REFERENCES}

[1] J.P. Aubin and I. Ekeland, Applied nonlinear analysis (John Wiley and Sons, New York, 1984).

[2] F.E. Browder, 'A new generalization of the Schauder fixed point theorem', Math. Ann. 174 (1967), 285-290.

[3] F.E. Browder, 'The fixed point theory of multi-valued mappings in topological vector spaces', Math. Ann. 177 (1968), 283-301.

[4] X.P. Ding, W.K. Kim and K.-K. Tan, 'Equilibria of non-compact generalized games with $L^{*}$-majorized preference correspondences', J. Math. Anal. Appl. 164 (1992), 508-517.

[5] B. Halpern, 'Fixed point theorems for set-valued maps in finite dimensional spaces', Math. Ann. 189 (1970), 87-98.

[6] P. Hartman and G. Stampacchia, 'On some nonlinear elliptic functional differential equations', Acta Math. 115 (1966), 271-310.

[7] C. Horvath, 'Some results in a multivalued mappings and inequalities without convexity', in Nonlinear and convex analysis: Lecture Notes in Pure and Appl. Math. Series 107 (Springer-Verlag, Berlin, Heidelberg, New York, 1987).

[8] H. Kneser, 'Sur un theoreme fondamental de la theorie des jeux', C.R. Acad. Sci. Paris 234 (1952), 2418-2420.

[9] W. Rudin, Functional analysis (McGraw-Hill Inc., 1973).

[10] M.-H.Shih and K.-K.Tan, 'Minimax inequalities and applications', Contemp. Math. 54 (1986), 45-63.

[11] M.-H.Shih and K.-K.Tan, 'Covering theorems of convex sets related to fixed point theorems', in Nonlinear and convex analysis, Editors B.L. Lin and S.Simons, pp. 235-244 (Marcel Dekker, 1987).

[12] K.-K. Tan, 'Comparison theorems on minimax inequalities, variational inequalities and fixed point theorems', J. London Math. Soc. 28 (1983), 555-562.

Department of Mathematics Education Chungbuk National University Cheongju 360-763

Korea
Department of Mathematics, Statistics and Computing Science Dalhousie University

Halifax NS

Canada B3H 3J5 